\title{
APROXIMACIONES AL ESTUDIO DEL CUERPO DEL DOCENTE
}

\author{
APPROACHES TO STUDYING THE BODY OF THE TEACHER
}

APROXIMAÇÕES AO ESTUDO DO CORPO DO DOCENTE

\section{Raimundo Villalba Labrador ${ }^{1}$}

\section{Resumen}

\begin{abstract}
En este artículo se revisa un conjunto de estudios que han contribuido en la indagación del cuerpo del docente en la educación escolar, desde diferentes abordajes (teóricos y metodológicos). Esta selección entrevé compresiones distintas del cuerpo del docente en sus múltiples facetas y experiencias, al considerar la docencia como una profesión corporal, subjetiva y situada en un contexto sociocultural. Dicho de otra manera, el cuerpo asume roles en los diversos escenarios en que se puede apreciar o vivenciar la labor docente. De aquí en adelante, se pueden diferenciar los tipos de hallazgos sobre el cuerpo docente, organizados de acuerdo con su objeto de estudio, las metodologías empleadas, las teorías del cuerpo abordadas y los resultados encontrados. Estos abordajes develan un campo de estudio para la investigación educativa, desde una aplicabilidad interdisciplinar que emerge tímidamente, y revelan posibilidades de exploración. Por lo anterior, preguntarnos por el cuerpo del docente contribuirá a comprender lo que supone la profesión, la enseñanza, la educación y el sujeto docente.
\end{abstract}

Palabras clave: cuerpo del docente; corporeidad y corporalidad; escuela; educación

\section{Abstract}

This paper reviews a compilation of research literature that has contributed to examine the teacher's body from different perspectives (both theoretical and methodological). This selection allows us to display different insights of the teacher's body in their multiple traits and experiences when considering teaching as a bodily, subjective profession located in a sociocultural context. In other words, the body adopts roles in the various scenarios where the teaching practice can be observed or experienced. From here on, the types of findings about the teacher's body can be differentiated, organized according to their object of study, the methodologies used, the theories of the body addressed, and the results found. These approaches reveal a field of study for educational research, from an interdisciplinary applicability that emerges timidly, and reveal possibilities for exploration. Therefore, asking ourselves about the body of the teacher will contribute to understanding what the profession, teaching, education and the teaching subject entails.

Keywords: teacher's body; corporality and corporeality; school; education

1 Licenciado en Humanidades y Lengua Castellana; especialista en Educación Artística; magíster en Estudios Artísticos; docente de la Secretaría de Educación Distrital. Estudiante del Doctorando en Artes y Ciencias del Arte LLA-CRÉATIS, de la Université de Toulouse Jean Jaurès, UT2J, y del Doctorado Interinstitucional en Educación DIE, de la Universidad Distrital Francisco José de Caldas, con énfasis Lenguaje y Educación. Orcid: 0000-0003-3978-0617. Correo electrónico: raimundovillalba@gmail.com 


\section{Resumo}

0 artigo revisa um conjunto de estudos que contribuem na indagação sobre o corpo do docente, no contexto da educação escolar, a partir de diferentes abordagens (tanto teóricos como metodológicos). Essa seleção de textos permite refletir sobre distintos conceitos do corpo do docente em suas múltiplas facetas e experiências ao considerar o ensino como uma profissão corporal, subjetiva situada num contexto sociocultural. Em outras palavras, o corpo assume papéis nos diferentes cenários onde pode-se valorar ou vivenciar o trabalho do docente. A partir daqui, pode-se diferenciar os tipos de material bibliográfico sobre o corpo docente organizados de acordo com seu objeto de estudo, as metodologias utilizadas, as teorias do corpo abordadas e os resultados encontrados. Essas abordagens revelam um campo do estudo para a pesquisa educacional a partir de uma aplicabilidade interdisciplinar que emerge timidamente e revelam as possibilidades de exploração. Portanto, perguntarmos pelo corpo do docente contribuirá a compreender o que supõe a profissão, o ensino, a educação e o sujeito docente.

Palavras-chave: corpo do docente; corporeidade e corporalidade; escola; educação

Fecha de recepción: 28 de enero de 2019

Fecha de evaluación: 18 de febrero de 2019

Para citar este artículo:

Villalba Labrador, R. (2019). Aproximaciones al estudio del cuerpo del docente. Lúdica Pedagógica, 29, 11-22. https://doi.org/10.17227/ludica.num29-11079 
La pregunta por el cuerpo del docente se ancla al estudio del cuerpo y su corporalidad, desarrollado por la comunidad académica latinoamericana desde la década de los ochenta (Rico, 1998; Castillo, 2015). Sus esfuerzos han consolidado publicaciones y espacios de intercambios de saberes ${ }^{2}$, con el objetivo de reflexionar y comprender, desde diferentes disciplinas y abordajes, el rol y la experiencia del cuerpo y las corporalidades en distintos ámbitos de la vida social y cotidiana en Latinoamérica (Castillo, 2015). Entre estos abordajes, el campo de la educación se ha enriquecido de diversas reflexiones que cuestionan el sistema educativo y la función histórica de la escuela en el modelamiento del cuerpo del educando. Al relacionar algunos puntos de la discusión, se puede indicar: primero, la función de la escuela en el modelamiento del cuerpo del educando y la formación de un sujeto dócil y subordinado, a partir de los fundamentos teóricos de Michel Foucault y Pierre Bourdieu, entre otros (Cabra y Escobar, 2014). Segundo, la comprensión de los modos históricos de civilización de los cuerpos en la consolidación de los Estados nación latinoamericanos, a través de la escuela (Pedraza, 2011; Martínez, 2012). Tercero, la marginación del cuerpo en el currículo escolar, reducido a la clase de educación física (Arboleda, 1997; Pedraza, 2011), cuyas prácticas corporales, centradas en la producción y la competencia, han sido fuertemente criticadas, y están en tratamiento de resignificación. Cuarto, los aportes de la pedagogía crítica (McLaren, 1994; 1997; 2005), cuyos esfuerzos apelan por una educación crítica, sociocultural y política de los cuerpos. De una manera u otra, el cuerpo del estudiante ha sido objeto de estudio y detonador de variadas reflexiones de la vida en la escuela, que han contribuido a proponer nuevas relaciones y prácticas corporales en el contexto escolar para la formación de un sujeto acorde a nuestra realidad latinoamericana.

Sin embargo, la atención académica en el estudio del cuerpo del docente es aún incipiente, no solo en Colombia (Cabra y Escobar, 2014; Villalba, 2015), sino también en Latinoamérica (Arévalo e Hidalgo, 2015), Inglaterra (Sparkes, 1996) y España (Martínez

2 Por ejemplo, el giro corporal, en Colombia; el Encuentro Latinoamericano de Investigadores sobre Cuerpos y Corporalidades en las Culturas, el Congreso Internacional El Cuerpo en el Siglo xxI: Aproximaciones Heterodoxas desde América Latina, entre otros. y González, 2016). Como sugieren Martínez y González (2016), son pocos los estudios que abordan la docencia como una profesión corporal, lo que refleja la ausencia del cuerpo en la investigación educativa y en la formación inicial y permanente del profesorado. De ahí que Sparkes (1996) enfatiza en la necesidad de estudiar los cuerpos subjetivamente experimentados, al reconocer que el cuerpo hace parte del yo de los docentes, es decir, "su género, edad, clase social, capacidad física, identidad sexual, raza, etnicidad, cansancio, estrés y reacciones emocionales se muestran en los distintos episodios escolares, sucesos críticos o momentos de desmoralización" (p. 101). Lo anterior sugiere un terreno fértil por explorar en perspectivas, aspectos y posibilidades metodológicas para interrogar la condición corporal docente. Comprenderlo nos puede revelar el sentido social escolar que impera en el docente, en quien las condiciones somáticas cobran uno u otro valor (Pedraza, 2009).

Con la intención de proyectar el terreno, el artículo revisa un conjunto de estudios que han contribuido en la indagación del cuerpo del docente en el contexto escolar, desde diferentes abordajes — teóricos y metodológicos- Los productos revisados (tesis de maestría y doctorado, publicaciones en revistas académicas, investigaciones en centros de estudios y laboratorios de universidades, entre otros) prestan atención a las experiencias corporales de los estudiantes en formación docente; a la subjetividad e identidad de los docentes en ejercicio; al aspecto psicomotriz del cuerpo; a la regulación institucional del cuerpo; y a la afectación somática laboral. Sin embargo, los abordajes revisados no siempre toman el cuerpo como objeto de estudio, sino que, en algunos casos, se refieren a aquel para reflexionar sobre aspectos específicos de la labor docente. De uno u otro modo, estos abordajes develan un campo de estudio para la investigación educativa, desde una aplicabilidad interdisciplinar que emerge tímidamente y que revela posibilidades de exploración. De aquí en adelante, se pueden diferenciar los tipos de hallazgos sobre el cuerpo docente, organizados según su objeto de estudio, las metodologías empleadas, las teorías del cuerpo abordadas y los resultados encontrados.

Para comenzar, con relación al objeto de estudio, se identificaron trabajos con objetivos de formación 
inicial del profesorado 3 . Por ejemplo, Castrillón (2016), de la Universidad de Antioquía (Colombia), sistematizó la experiencia de la asignatura Pedagogía del Cuerpo, realizada en una institución normalista ${ }^{4}$ para que los estudiantes en formación reflexionen sobre el cuerpo del docente antes de ejercer la profesión. Con este propósito, la investigadora aborda la literatura sobre el cuerpo para establecer analogías con el cuerpo del docente en la acción pedagógica. Esta analogía permite reflexionar sobre la acción docente, tanto de la docente investigadora, como de los estudiantes, al hacerlos partícipes de sus experiencias personales. En esta misma línea de formación inicial del profesorado, encontramos abordajes autobiográficos (Marcos, 2012; Prats, 2013; Albarrán, 2016) realizados en el Programa de Investigación y Formación de la Universidad de Valladolid (España), liderado por los investigadores Martínez y González (2016), quienes estudian el cuerpo y la dimensión corporal del docente a partir de las experiencias de los estudiantes docentes en formación. Para este propósito, utilizan las autobiografías corporales y los diarios corporales docentes para registrar su experiencia y analizarla según cuatro categorías de observación de la dimensión corporal del docente: 1) salud y energía, 2) emociones, 3) comunicación y relaciones y 4) imagen corporal. El propósito de este proyecto es "contribuir a la escasa literatura sobre la importancia de dar luz a las experiencias corporales de los futuros docentes e ilustrar las interconexiones que estas experiencias tienen entre sí y con el desarrollo de su identidad profesional" (p. 260).

Por otra parte, se identificaron trabajos interesados por la relación cuerpo y subjetividad de los docentes. Entre estos, Arévalo e Hidalgo (2015), de la Universidad Nacional de Chile, comprenden las subjetividades del profesorado escolar chileno, a través de la práctica corporal y la escritura narrativa como metodologías para mostrar dicha relación. Los docentes participantes valoran y visibilizan su cuerpo como herramienta de aprendizaje, expresión, comunicación y creación. Otro ejemplo es el estudio realizado por Tarruella y Rodríguez (2008), de la Universidad

3 Referido a la formación de los estudiantes en facultades de educación o normales para devenir futuros docentes.

4 Instituciones encargadas de la formación inicial de los maestros de preescolar y básica primaria en el sistema educativo colombiano.
Nacional del Comahue (Argentina), quienes observaron la construcción de un modo de ser cuerpo en los docentes, a partir de la repetición de un conjunto de prácticas corporales que suceden en las instituciones educativas, y que son reconocidas y validadas socialmente. En su estudio, argumentan que la escuela se convierte en un lugar privilegiado para la delimitación de lo corporal, al establecer prácticas y hábitos específicos que los sujetos encarnan; en otras palabras, los docentes reproducen significados de relaciones hegemónicas predominantes a través del cuerpo. En esta misma lógica, Bolívar, Castro, Díaz y Peñaloza (2015), de la Universidad Pedagógica Nacional (Colombia), describen la manera en que las instituciones educativas configuran la corporalidad y la corporeidad del docente, a través de las dinámicas de regulación institucional, tras observar la relación de estas con el lenguaje corporal, los modos de vestir y las proyecciones laborales de cada docente. Sparkes (1996), de la Universidad de Exeter (Inglaterra), analiza el modo como algunos docentes de Educación Física afrontan las interrupciones de sus proyectos profesionales a causa de limitaciones corporales por vejez, enfermedad o lesión. Las narrativas citadas revelan la relación cuerpo e identidad, en la cual los cambios corporales modifican el yo. Finalmente, Villalba (2015), de la Universidad Distrital (Colombia), utiliza la escritura autoetnográfica para preguntarse por su condición corporal como docente, a partir del reconocimiento de las marcas que esta experiencia ha dejado en su cuerpo.

En lo referido a cuestiones didácticas, se relacionan estudios centrados en el papel del cuerpo en la actividad de la enseñanza, en los grados de preescolar o primera infancia. Desde la categoría disponibilidad corporal, las investigadoras argentinas Rodríguez, Arias y Uría (2014) observan el papel del cuerpo del docente en el despliegue psicomotor de los niños de tres años, al comprender su influencia en el aprendizaje desde una perspectiva psicomotriz. En el mismo nivel educativo, Páez (2008) enfatiza en la importancia del cuerpo de la docente de preescolar para acompañar la formación de los niños. Por lo anterior, establece cuatro categorías de la acción corporal pedagógica: 1) protector, 2) sin protección, 3) en movimiento y 4) sujetador. Por lo tanto, la cercanía corporal de la profesora con el niño es vital, puesto que resulta imposible una formación desde el tablero 
o solo desde el discurso. Finalmente, se identificaron una amplia variedad de publicaciones que abordan el cuerpo del docente desde la patología de la enfermedad profesional (Arón y Milicic, 2000; Padilla et al., 2009; Alves y Nuño, 1996; Fernández, 2014), a través de disciplinas como la Psicología, la Salud Ocupacional y la Medicina. Aparece, entonces, el estudio del cuerpo de los docentes, manifestado en su desgaste corporal, el dolor, la lesión o la afectación psicológica.

Con relación a los diseños metodológicos, estos se ajustan a los propósitos de la investigación y a la perspectiva del cuerpo teorizada por los investigadores. Entre estos, se observa una alta tendencia hacia la investigación de perspectiva biográfica y narrativa, para los trabajos interesados en los cuerpos subjetivamente experimentados; $y$ una tendencia etnográfica, para las investigaciones de perspectivas objetivas, que privilegian la observación de campo y las entrevistas. De esta manera, el primer grupo relaciona experiencias que implican talleres de práctica corporal y escritura narrativa biográfica (Arévalo e Hidalgo, 2015; Castrillón, 2016), o ejercicios relacionados con el enfoque fenomenológico hermenéutico, a partir de los abordajes teóricos de Merleau Ponty y Husserl (Páez, 2008; Castrillón, 2016). Otras utilizan las historias de vida y las autobiografías, a través de entrevistas, registros de relatos, viñetas narrativas y anécdotas, para indagar por las experiencias personales (Marcos, 2012). Asimismo, algunos autores proponen otras rutas creativas y narrativas para recuperar la experiencia del cuerpo. Villalba (2015), por ejemplo, propone el corporrelato como una práctica analítica y creativa que contribuye a la producción de conocimiento del cuerpo desde la experiencia del docente investigador. Asimismo, Martínez y González (2016), de la Universidad de Valencia, utilizan las autobiografías corporales y los diarios corporales docentes como herramientas para registrar las experiencias docentes en las que el cuerpo pasa al primer plano.

El diario corporal docente es un híbrido entre un diario docente (aquel en el que recogemos por escrito lo que nos ocurre como profesores para tener datos y relatos con los que poder reflexionar y replantear la docencia) y un diario corporal (aquel centrado en los momentos en los que, por diferentes motivos, el cuerpo "pasa a primer plano" y "escuchamos" al cuerpo, lo que nos permite tomar conciencia de nuestra naturaleza corpórea) (Albarrán, 2016, p. 10).

En contraste, los estudios interesados en observar la dimensión corporal con mayor distancia entre el investigador y el objeto de estudio (Rodríguez et al., 2014; Bolívar et al., 2015) utilizan la etnografía y diversas técnicas de recolección de datos - como observación participante, fichas de observación, registros en diarios, registros de videos, encuestas y entrevistas grupales - para mostrar el cuerpo en acción.

El interés se ha centrado en observar, relacionar, caracterizar e interpretar la corporalidad y la corporeidad de los docentes a partir del lenguaje corporal, los modos de vestir, los gestos, los silencios, el contacto, las miradas, etc., y lo invisibilizado por la institución educativa, para buscar huellas incorporadas de la regulación institucional (Bolívar et al., 2015. p. 4).

Preguntarse por el cuerpo no predispone una mirada hegemónica u homogénea del mismo, sino que las posibilidades de su estudio responden también a diversas posturas teóricas que se relacionan con su experiencia o prestan atención en una u otras dimensiones. Por lo tanto, el cuerpo alberga una polisemia de significados que varían en relación con el contexto y la perspectiva del estudio (Rico, 1998; Cabra y Escobar, 2014; Pedraza, 2009). Dada esta posibilidad, las perspectivas teóricas utilizadas en el estudio del cuerpo del docente transitan entre la filosofía, la educación, la sociología y la antropología, en algunos casos con la intención de sobrepasar la concepción cartesiana y dualista del ser humano, "cambiar esa concepción del racionalismo puramente instrumental dentro de las escuelas y promover otros modos de estar-en-el-mundo, empezando por prestar atención al propio cuerpo del educador" (Martínez y González, 2016, p. 261). En esta polisemia, cabe destacar el papel fundamental de la fenomenología, derivada de autores como Husserl y Merleau Ponty, al proponer las categorías cuerpo Leib, cuerpo Körper y Quiasmático (Gallo, 2006), las cuales han ampliado las posibilidades de acercarse al estudio del cuerpo desde el cuerpo vivido o el cuerpo encarnado. En otra línea, desde algunas posturas sociológicas, los investigadores citan a Le Breton para referir a la categoría corporeidad y su relación con la subjetividad, y a Bourdieu 
con su categoría habitus (Rodríguez, et al., 2014; Martínez y González, 2016; Tarruella y Rodríguez, 2008). "Este habitus nos da esa idea de cuerpo como bisagra entre la voluntad de la persona y la incorporación de las restricciones y situaciones culturales y contextuales" (Bourdieu, 1998); "se configura como el modo de obrar, pensar y sentir asociados a la posición social, a cómo el sujeto percibe el mundo y cómo actúa (corporalmente) en él” (Martínez y González, 2016, p. 262).

Desde una argumentación teórica interdisciplinar, Sparkes (1996) posiciona el cuerpo como el principal símbolo del yo, razón por la cual los cambios corporales lo modifican. Por lo tanto, ser persona implica corporeidad, es decir, estar vinculado a un cuerpo. A su vez, Tarruella y Rodríguez (2008) utilizan la categoría de la encarnación, a partir de los fundamentos teóricos de la pedagogía crítica de McLaren, para considerar el cuerpo como:

Un 'cuerpo/sujeto', es decir, como el terreno de la carne en el que se inscribe, se construye y se reconstituye el significado. Desde esta perspectiva, el cuerpo se entiende como la 'encarnación' de la subjetividad, que también refleja la sedimentación ideológica de la estructura social inscripta dentro de ella [...]. Los cuerpos/sujetos muestran lo encarnado, en su piel, en sus músculos, en sus huesos, en sus posturas, en sus gestos, en su andar, en sus malestares, en sus enfermedades (p. 1).

Es necesario señalar la tendencia a utilizar las categorías de corporalidad y corporeidad para aproximarse al cuerpo y a la subjetividad. Sin embargo, los significados difieren según los autores abordados y la perspectiva de su estudio. Por ejemplo, Bolívar et al. (2015) define la corporalidad como una construcción física y biológica que surge de nuestra existencia (Körper), y la corporeidad (Leib), como la proyección simbólica que hacemos de nuestro cuerpo, de nosotros y de nuestra existencia. En cambio, Marcos (2012) observa la corporeidad desde una perspectiva no solo biológica del cuerpo, sino también "como nuestra forma de estar en el mundo, de percibirlo y de relacionarnos con él; va a estar presente en toda nuestra vida, aunque siempre en continuo cambio y transformación" (p. 9). Mientras tanto, Arévalo e Hidalgo (2015) utilizan la categoría de corporeidad para señalar la relación imbrica entre cuerpo, sujeto y cultura, a partir de autores como Cachorro, Merleau Ponty y McLaren, lo que da cuenta de la encarnación. Por lo tanto, comprenden la docencia como una experiencia corporal y subjetiva en donde el cuerpo, citando a Gallo, se concibe como "el lugar donde se inscriben, construyen y reconfiguran los acontecimientos del existir del docente en la escuela" (p. 167).

Para algunos investigadores, asumir una sola perspectiva de cuerpo o concepto no es suficiente para sus propósitos investigativos. En consecuencia, elaboran cruces teóricos de diferentes autores, en especial contemporáneos, para ampliar la comprensión del cuerpo. Tal ejemplo se observa en Martínez y González (2016), quienes proponen una visión holística del cuerpo del educador desde un enfoque poliédrico, determinado tanto por las experiencias y vivencias del educador, como por los contextos históricos y sociales. Según esto, el cuerpo es una construcción social fuertemente condicionada por la trayectoria de vida personal y por las circunstancias culturales y sociales en que está imbricado. Esta misma lectura se observa en Rodríguez et al. (2014), para definir la categoría disponibilidad corporal desde una perspectiva interdisciplinar. Los autores ponen en diálogo los aportes del psicoanálisis, la pedagogía y la psicomotricidad para observar esta dimensión del cuerpo del docente y su mediación en el aprendizaje motriz de los niños en su primera etapa de vida. Asimismo, Villalba (2005) propone un diálogo entre autores para teorizar la relación entre cuerpo, experiencia y marcas corporales. Para este propósito, cita autores como Rico Bovio, Larrosa, Mandoki y Myss para sostener que la experiencia del docente se va marcando en su cuerpo. Por lo tanto, preguntar por las marcas es preguntar por las experiencias, y en las experiencias se revela el cuerpo.

Dentro de los resultados y conclusiones de estos estudios sobre el cuerpo de los docentes, es evidente la consciencia corporal de los docentes participantes, durante y después del proceso (Arévalo e Hidalgo, 2015; Castrillón, 2016; Martínez y González, 2016). Esto le permitió elaborar reflexiones sobre lo vivido y las posibilidades de transformación de la experiencia corporal en la práctica pedagógica y en el sujeto. Asimismo, se reconoce que la negación y el control que la escuela ejerce sobre los cuerpos no solo se limitan a los estudiantes, sino que incluyen también 
los cuerpos de los docentes. En otras palabras, sus cuerpos rígidos controlan y vigilan, al tiempo que son objeto de control y vigilancia por la comunidad escolar. Esta manera de vivenciar el cuerpo se denomina, según Tarruella y Rodríguez (2008), "usos ordenados del cuerpo de los/as docentes", los cuales se aprenden y sostienen en la labor docente, no solo a través del orden discursivo (saber-poder), sino también a través de rituales corporales y organización corporal, es decir, de la repetición de las posturas habituales del cuerpo, como la resultante del conjunto de prácticas estereotipadas. Estos rituales se observan en:

[...] la maestra de brazos cruzados escuchando las excusas de algún alumno que no hizo la tarea; la docente parada en el medio y al final de las filas de varones y mujeres; tener el rostro serio imponiendo respeto. La forma de pararse para explicar un tema; estar siempre de pie, nunca sentados; gesticular cuando se habla; colocar las manos detrás o tomarlas adelante; el tono de voz que se usa y la presencia corporal activa en el cuidado de los recreos (p. 2).

Los rituales corporales, al ser encarnados por lo docentes, conllevan un significado de legitimidad y validación social en el entorno escolar para sostener una determinada ideología dominante que contribuye al borramiento del cuerpo/sujeto, tanto de los docentes, como de los estudiantes. En consecuencia, configuran un modo de subjetividad encarnada de significaciones sociales y culturales dominantes. Por otro lado, Bolívar et al. (2015) han mostrado que los docentes incorporan aspectos corporales relacionados con la disciplina de enseñanza, la normatividad de la institución y la infraestructura del centro escolar. Por lo tanto, el cuerpo es un texto por interpretar de acuerdo con su realidad sociocultural. De esta manera, los docentes de cultura, los de primaria, los de bachillerato y niveles superiores difieren en su expresividad, movimientos, comportamientos, maneras de vestir, discurso, perspectivas profesionales, uso de los espacios y desplazamientos en la institución educativa... aspectos relacionados con las necesidades pedagógicas y didácticas de la enseñanza, las condiciones reguladoras de la institución y su identidad docente. Por ejemplo, en el desarrollo de las clases, los docentes de primaria realizan más movimientos que sus colegas de bachillerato, lo que les demanda un vestuario confortable y el uso de la bata para realizar sus actividades. Esta situación no preo- cupa a los de secundaria. Otro ejemplo es el docente de cultura, para quien su cuerpo y su movimiento es parte de la enseñanza. Por lo tanto, la vestimenta y la expresividad cobra sentido disciplinar. Entre la diversidad de aspectos descritos sobre la corporeidad y la corporalidad del docente, los investigadores concluyen que la noción de institucionalidad revela la escuela como un lugar cerrado y con normas predeterminadas, donde los docentes se relacionan a través de reglas, convenciones, derechos, hábitos y costumbres para seguir un objetivo común.

De manera análoga, Arévalo e Hidalgo (2015) resaltan la importancia del cuerpo del docente en la construcción de vínculos afectivos con los estudiantes que contribuyen en los procesos de aprendizaje, pues "las emociones están antes y después del conocimiento cognitivo" (p. 169). Sin embargo, estos modos de relación son deslegitimados institucional y socialmente en la labor docente, lo que limita la experiencia corporal en las relaciones pedagógicas.

Y lo otro es esta cosa de ser como rígida. Hoy en día yo soy bastante afectiva en cuanto a mis alumnos... pero... con todo este tema de la pedofilia, se nos indica que, por favor, si un alumno te va a abrazar dile que no.... Entonces, es súper chocante esta cosa de la afectividad (p. 170).

En cuanto al trabajo desarrollado en la Universidad de Valladolid (Martínez y González, 2016; Marcos, 2012; Prats, 2013), interesados en las primeras experiencias de la profesión docente de los estudiantes en formación, identificaron cuatro grandes ámbitos en los que la dimensión corporal del docente está particularmente presente: 1) salud y energía, 2) emociones, 3) comunicación y relaciones y 4) imagen corporal. Con relación al ámbito de salud y energía, observan que la exigencia física y energética del cuerpo para el desempeño de la labor docente varía según su nivel y área de enseñanza. De esta manera, si las condiciones físicas y energéticas requeridas para un desempeño específico no se proporcionan de manera adecuada, son causas de inhabilitación de la acción docente, a través de la incapacidad laboral o el cambio de área ${ }^{5}$. El ámbito de la comunicación

5 Por ejemplo, en el caso de los docentes de educación física, en algunos países, una limitación física podría ser causa de cambio de área: pasar de especialistas en educación física a enseñar en el aula como tutores o maestros de Lenguaje o de Matemáticas (Martínez y González, 2016). 
corporal refiere a las actividades físicas, artístico-expresivas, rituales, de expresión disciplinaria y a la mirada. También incluye los momentos en que la relación pedagógica necesita un acompañamiento o modelo corporal (como en las áreas de currículo corporal o en la primera infancia), para que los alumnos aprendan por observación o imitación.

Nos referimos, también, a cómo el docente puede incorporar y transmitir el gusto y la pasión por lo que enseña y mostrar el vínculo que le une al grupo y a cada estudiante. El cuerpo es un aspecto central para dar vida a lo que se enseña, para encarnar el valor de lo que queremos que los estudiantes aprendan (p. 268).

En el ámbito de sentimientos y emociones, observan las diferentes emociones y sentimientos, tanto negativos como positivos, incorporados en la labor docente y devenidos de diversas experiencias pedagógicas y sociales con la comunidad escolar. Por ejemplo:

[...] la inseguridad que un profesor novel puede tener en sus primeros contactos con la clase se traduce en sensaciones en el estómago, sequedad en la boca, tics, inquietud general, sensación de que no sabes dónde poner las manos, refugiarse detrás de la mesa del profesor, temblor de manos, taquicardia, ahogo (Marcos, 2012, p. 22).

En el ámbito esquema o imagen corporal, los autores revelan la proyección de la presencia corporal del docente en la escuela y sus propósitos pedagógicos. Su manera de vestir, el maquillaje, los accesorios que utiliza, la barba, el sudor, la ubicación en el salón, su proximidad al compartir con los estudiantes... son aspectos percibidos por su comunidad. Por ejemplo, se puede promover, sin desearlo, una imagen de consumo cuando el docente viste y porta accesorios de moda. Aspectos que los estudiantes no pasarán desapercibidos (Albarrán, 2016). Para los estudiantes en formación docente, este ámbito es fundamental en sus primeras prácticas, puesto que, en su objetivo de ser reconocidos como docentes por la comunidad escolar, deben reflexionar sobre el equilibrio entre los rasgos de la identidad docente y los propios (Martínez y González, 2016). Finalmente, en el énfasis de educación física, utilizan el estudio de los relatos de vida para comprender cómo los hitos de la experiencia corporal de los estudiantes fueron fundamentales en la configuración de su identidad docente (Marcos, 2012; Prats 2013; Martínez y Gonzalez, 2016).

Sparkes (1996), al estudiar los relatos de vida de docentes de educación física, observó que proyectos corporales interrumpidos, comúnmente relacionados con accidentes, enfermedades o envejecimiento, influyen en su subjetividad tras cambiar y transmutar el modo en que ellos construyen narraciones e identidades de sí mismos y de su profesión. Estas limitaciones corporales se distancian del yo profesor como cuerpo idealizado y configurado en una sociedad de consumo. Por consiguiente, los docentes prefieren realizar cambios en su vida profesional para evitar la frustración de un "yo no deseado" y reconstruir otra identidad en torno al cuerpo con el que realmente viven o vivirán. El investigador encontró que las narrativas analizadas tienen sus raíces en discursos dominantes, disponibles en la cultura occidental, y que limitan el potencial para explorar y cambiar el sentido del yo. Citando a Denzi, el autor agrega: "ningún relato de una experiencia personal es jamás una producción individual. Emana del grupo más amplio, del contexto cultural, ideológico e histórico" (p. 115).

Con respecto a Villalba (2015), en su trabajo autoetnográfico de escritura creativa desde el cuerpo (corporrelato), creó una serie de relatos que ilustran la experiencia de ser cuerpo docente en la escuela. Estos relatos se analizaron según seis categorías devenidas de la afectación corporal y características de la experiencia: 1) cuerpo vulnerable, 2) cuerpo desgastado, 3) cuerpo autoritario, 4) cuerpo silenciado, 5) cuerpo creativo y 6) cuerpo cuidador. El cuerpo vulnerable revela el miedo, la frustración profesional y la indolencia como marcas de experiencias vividas en situaciones de amenazas a docentes o atraco con arma blanca o de fuego. El cuerpo desgastado revela las marcas en el cuerpo del docente a causa de su actividad profesional: problemas de voz, dolores musculares, agotamiento físico y energético, estrés laboral y ansiedad son las marcas identificadas en los corporrelatos que revelan la condición corporal de desgaste laboral. El cuerpo autoritario revela las manifestaciones fisiológicas que se activan inconscientemente en el cuerpo del docente como gestos de autoridad: la exaltación corporal, la mirada vigilante o de disgustos, las arrugas faciales, la coloración facial, el regaño y el grito son las marcas de este cuerpo para regular las dinámicas corporales 
de los estudiantes en la escuela. El cuerpo silenciado revela las tensiones de una construcción de identidad corporal diferente a la estructura heterosexual normalizada que promueve la escuela. Así, la incorporación de comportamientos estereotipados del género masculino, el miedo a la etiqueta, el señalamiento y la exclusión son las marcas de este cuerpo para ser imperceptible en el contexto escolar. El cuerpo creativo revela las marcas devenidas de experiencias de creación en el cuerpo del docente. La creatividad del docente está puesta en las posibilidades de transformar, didáctica o pedagógicamente, las experiencias de la comunidad escolar y las propias para resignificar su ser/hacer profesional. En el caso de Villalba, el cuerpo creativo lo compone la memoria sensible como docente de artes en los procesos de creación artística en danza y comparsa con sus estudiantes y la comunidad. Finalmente, el cuerpo cuidador reflexiona sobre el cuidado del sí y de los otros (estudiantes), como modos de relación sensible que ha encarnado el docente al vivenciar experiencias de accidentes donde la vida se pone en riesgo. Estas memorias se van encarnando como marcas que se activan para responder en casos de emergencia escolar.

En relación con lo didáctico, Rodríguez et al. (2014) manifiestan la carencia de producción académica en la implicación del cuerpo en el proceso de enseñanza y aprendizaje en el campo de la psicomotricidad en la educación inicial. Al parecer, el cuerpo del docente carece de relevancia en la formación profesional y en el diseño curricular de la práctica docente. Por lo anterior, las investigadoras analizan la categoría disponibilidad corporal para observar y describir las formas en que se manifiesta el cuerpo del docente en los distintos escenarios escolares, con el objetivo de identificar prácticas y actitudes que favorecen u obstaculizan el singular funcionamiento psicomotor de los niños. Entre los indicadores de observación de la categoría, las autoras incluyen "capacidad de receptividad, capacidad de escuchar, capacidad para observar, expresión de la mirada, expresión de la voz, uso de la palabra, gestualidad, resonancias tónico-emocionales, entre las más significativas" (p. 6). Con los mismos intereses, Páez (2008) reflexiona sobre la importancia del cuerpo de la profesora de preescolar en el vínculo afectivo y formativo de sus estudiantes, así como en su autoformación docente. En palabras de la autora:
¿Qué hace distinto al cuerpo de la maestra de preescolar? Lo diferente es la forma como, en su relación con los niños que han sido puestos a su cuidado y formación, este experimenta la necesidad de proteger, de ayudar y de guiar en un acercamiento corporal mutuo que contribuya a tales fines. Lo que pasa por el cuerpo de la docente va dejando en ella un aprendizaje, una marca que será referente para futuras interacciones. Los niños necesitan cercanía, presencia física, la corporalidad de la maestra para conocer; el cuerpo de la docente requiere lo mismo para enseñar (p. 27).

Por lo anterior, la autora propone cuatro modos de socialización y de cercanía en el acompañamiento de los niños de preescolar. Un cuerpo protector, de naturaleza instintiva y protectora de la mujer y su rol de madre. Un cuerpo sin protección, expuesto a los contactos físicos, ruidos, excreciones y expresiones biológicas, emocionales y afectivas de sus estudiantes. Un cuerpo sujetador, encargado de sujetar y mostrar los diferentes materiales y objetos del mundo a los niños. Un cuerpo en movimiento, dispuesto a la acción y a la calma, a ser modelo del aprendizaje corporal de los niños, a normalizar los cuerpos de los niños y a realizar todas las actividades necesarias del proceso escolar. En esta relación didáctica de los aprendizajes de preescolar, la proximidad del cuerpo de la maestra es vital para los estudiantes; de lo contrario, "resulta difícil —o mejor, imposible — una formación desde el tablero o solo desde el discurso. La formación se da con el cuerpo y no fuera de este" (Páez, 2008, p. 138).

En cuanto a estudios distintos al campo de la educación interesados por el cuerpo de los docentes, la medicina laboral lo analiza desde su condición patológica, a través de la Psicología, la Salud Ocupacional y la Medicina. Entre estos, se reconoce que la docencia es una de las profesiones más afectadas por el estrés laboral y el síndrome de burnout (Arón y Milicic, 2000; Padilla et al., 2009; Vidal, Nicasio y Pacheco, 2010). Un estudio realizado en Colombia, con 367 docentes de tres colegios públicos en Bogotá, destacó que, por lo menos, el 15,6 \% de los docentes estudiados presentaban síndrome de burnout (Padilla et al., 2009), lo cual constituye un indicador importante para la salud mental de los docentes. Por ello, numerosos estudios (De la Torre, 2007) se interesan por indagar las condiciones de las causas y las manifestaciones de este mal, así como su impacto en 
la comunidad escolar y la labor docente, además de acciones preventivas y terapias de intervención clínica. Por otro lado, diversos estudios se centran en los factores de riesgo que influyen en la aparición de trastornos de la voz en el personal docente, como la disfonía y los nódulos vocales (Preciado y Pérez, 2003; Alves y Nuño, 1996).

Fernández (2014) relaciona los riesgos laborales a los que se expone la profesión docente a partir de la literatura sobre el tema. Los resultados están clasificados en dos rangos de afectación: riesgos físicos, como enfermedades infecciosas (gripe, catarro, parasitarias, etc.); problemas de la voz; afecciones cardiovasculares; problemas musculoesqueléticos (lumbalgias, cervicales, fracturas, etc.); y riesgos psicosociales, como el estrés y el síndrome de burnout (o síndrome del quemado por el trabajo). Además, los resultados muestran que las afectaciones patológicas en la labor docente están asociadas a condiciones ambientales del entorno laboral, a la interacción que se expone el docente con agentes infecciosos, al uso de materiales cotidianos como la tiza, a las prácticas corporales repetitivas y cotidianas, a la utilización excesiva e inadecuada del cuerpo (como en el caso de la voz), a la manera cómo percibe las dificultades que presentan los estudiantes en los procesos de aprendizaje, a las demandas burocráticas de la labor, a la relación con los padres y los acudientes, al ambiente laboral, a las dificultades con los administrativos y a su subjetividad, es decir, a su edad, experiencia, carácter, entre otros factores.

Con el ánimo de ir cerrando este breve panorama sobre el cuerpo del docente, se puede decir que, desde la distancia del etnógrafo, quien observa los cuerpos en su ser/hacer docente, o desde los relatos de quienes han encarnado esta labor, es evidente que la docencia es una profesión corporal en la que el cuerpo asume un rol en los diversos escenarios en que se puede apreciar o vivenciar la labor docente. Desde esta comprensión, se proyectan múltiples posibilidades de indagar y ampliar el conocimiento sobre el cuerpo del docente, en especial, en aquellos aspectos inexplorados o apenas enunciados por los estudios abordados. Entre estas posibilidades de intervención, la carencia de estudios en el componente didáctico demanda con urgencia la reflexión sobre el lugar del cuerpo del docente en la acción didáctica, no limitada a su capacidad de enunciación o comunicación genérica, sino en la manera como el cuerpo hace parte del proceso de enseñanza ${ }^{6}$ del campo disciplinar y del nivel de formación.

Por otro lado, indagar por el cuerpo de los docentes revela nuevos aspectos para la comprensión de la profesión y lo que implica su encarnación en condiciones socioculturales específicas. Algunos de estos aspectos están relacionados con rasgos de la corporeidad subjetiva que influyen en la experiencia docente, es decir, la tensión entre un cuerpo subjetivo - parte del yo- y un cuerpo idealizado de la profesión. Por lo anterior, se hace necesario profundizar en los problemas relacionados con el género, la sexualidad, la vejez, la raza, las posibilidades corporales, etc., dado que el cuerpo de los docentes, como lo han indicado Martínez y González (2016), tiene una importancia notoria para comprender la enseñanza, la escuela, la educación y lo que supone la profesión.

\section{REFERENCIAS}

Albarrán, A. (2016). El diario corporal docente: una herramienta de reflexión, autoconocimiento y mejora en la formación inicial del profesorado de educación física. [Tesis de grado, Universidad de Valladolid]. Repositorio institucional, UVa. http://uvadoc.uva.es/ handle/10324/21059

Arboleda, R. (1997). Cuerpo y pedagogía. Educación Física y Deporte, 19(2), 83-91. https://aprendeenlinea.udea. edu.co/revistas/index.php/educacionfisicaydeporte/ article/view $/ 3787 / 3654$

Arévalo, A. e Hidalgo, F. (2015). Corporalidad docente: un abordaje para aproximarse a la subjetividad del profesorado del sistema escolar chileno. Revista Corpografías: Estudios críticos de y desde los cuerpos, 2(2), 162-179. http://dx.doi.org/10.14483/udistrital.jour. corpo.2015.1.a10

Arón, A. y Milicic, N. (2000). Desgaste profesional de los profesores y clima social escolar. Revista Latinoamericana de Psicología, 32(3). https://www.researchgate. net/publication/26595565_Desgaste_profesional_de_ los_profesores_y_clima_social_escolar

Alves, V. y Nuño, P. (1996). Problemas de la voz en el profesorado. Interuniversitaria de Formación del Profeso-

6 Lo didáctico se refiere a las metodologías y los conocimientos propios del campo disciplinar que son objeto de adaptaciones y transformaciones didácticas para la enseñanza (Bolívar, 2005, p. 4), así como a la pertinencia ética del conocimiento en la formación crítica del sujeto para una vida más humana y corporal. 
rado, (26), 33-42. https://dialnet.unirioja.es/servlet/ articulo?codigo $=117903$

Bolívar, A. (2005). Conocimiento didáctico del contenido y didácticas específicas. Revista de Currículum y Formación del Profesorado, 9(2). https://www.ugr.es/ recfpro/rev92ART6.pdf

Bolívar, O., Castro, J., Díaz, G. y Peñaloza, G. (2015). Configuración de la corporalidad y corporeidad del docente en la regulación institucional del colegio y la universidad. [Tesis de maestría, Universidad Pedagógica Nacional, Fundación Centro Internacional de Educación y Desarrollo Humano (Cinde)]. Repositorio institucional Cinde. https://repository.cinde.org. co/bitstream/handle/20.500.11907/1522/BolivarSilvaCastroCalaDiazRomeroPenalozaGarzon 2016. pdf? sequence $=1 \&$ isAllowed $=y$

Cabra, N. y Escobar, M. (2014). El cuerpo en Colombia: estado del arte y subjetividad. Bogotá: Instituto para la Investigación Educativa y el Desarrollo Pedagógico.

Castillo, S. (2015). Una mirada sobre el giro corporal en Colombia. Cuadernos de Música, Artes Visuales y Artes Escénicas, 10(1), 8-15. http://revistas.javeriana.edu. co/index.php/cma/article/view/13542

Castrillón, D. (2016). Pedagogía del cuerpo: una senda a la formación de maestros. [Tesis de maestría, Universidad de Antioquia] Repositorio institucional UdeA. http:// hdl.handle.net/123456789/2120

De la Torre, C. (2007). El malestar docente: un fenómeno de relevancia internacional. Docencia e Investigación. Revista de la Escuela Universitaria de Magisterio de Toledo, (17), 301-325. http://stellae.usc.es/red/file/ download/5625

Díaz, S., González, C. y Jaramillo, R. (2006). Aproximación a las problemáticas psicosociales y a los saberes y habilidades de los docentes del distrito. Revista de Estudios Sociales, (23), 45-55. http://www.scielo.org.co/pdf/ res/n23/n23a05.pdf

Fernández, P. (2014). Evaluación de la salud laboral docente: estudio psicométrico del cuestionario de salud docente. [Tesis de doctorado, Universitat Ramon Llull]. Repositorio institucional URL (en versalitas). http:// hdl.handle.net/10803/283976

Foucault, M. (2008). Vigilar y castigar: nacimiento de la prisión. Buenos Aires: Siglo XXI.

Gallo, L. (2006). El ser-corporal-en-el-mundo como punto de partida en la fenomenología de la existencia corpórea. Pensamiento Educativo, (38), 46-61.
Marcos, M. del V. (2012). Dimensión corporal de la profesión docente. [Tesis de grado, Universidad de Valladolid]. Repositorio institucional, UVa. https://uvadoc.uva.es/ bitstream/10324/2072/1/TFG-L\%20104.pdf

Martínez, A. (2012). Verdades y mentiras sobre la escuela. Bogotá: IDEP.

Martínez, L. y González, G. (2016). Docentes de carne y hueso: enseñar con cuerpo. Revista Ágora para la Educación Física y el Deporte, 18(3), 259-275.

Mclaren, P. (1994). Pedagogía crítica, resistencia culturaly la producción del deseo. Buenos Aires: Aique.

Mclaren, P. (1997). Pedagogía crítica y cultura depredadora. Políticas de oposición en la era postmoderna. Barcelona: Paidós.

Mclaren, P. (2005). La vida en las escuelas: una introducción a la pedagogía crítica en los fundamentos de la educación. Ciudad de México: Siglo XXI.

Padilla, A., Gómez, C., Rodríguez, V., Dávila, M., Avella, C., Caballero, A., ..., Hernández, S. (2009). Prevalencia y características del síndrome de agotamiento profesional (SAP) en docentes de tres colegios públicos de Bogotá (Colombia). Revista Colombiana de Psiquiatría, (38), 50-65. https://www.redalyc.org/articulo. oa?id=80615419005

Páez, R. (2008). El cuerpo de la maestra de preescolar y su papel en la formación de los niños. Revista Iberoamericana en Educación, (47), 123-139.

Prats, J. (2013). La dimensión corporal de la profesión docente. [Tesis de grado, Universidad de Valladolid]. Repositorio institucional UVa.

Pedraza, Z. (2011). En cuerpo y alma. Visiones del progreso y de la felicidad. Educación, cuerpo y orden social en Colombia (1830-1990). Bogotá: Universidad de los Andes.

Pedraza, Z. (2009). En clave corporal: conocimiento, experiencia y condición humana. Revista Colombiana de Antropología, (45), 147-168.

Preciado, C. y Pérez, J. (2003). Nódulos de cuerdas vocales. Factores de riesgos en los docentes: Estudios de casos y controles. Acta Otorrinolaringológica Española, (54), 253-260. http://www.elsevier.es/ es-revista-acta-otorrinolaringologica-espanola-102-articulo-nodulos-cuerdas-vocales-factores-riesgo-S000165190378412X

Rico, A. (1998). Las fronteras del cuerpo: crítica de la corporeidad. Quito: Abya-Yala. 
Rodríguez, M., Arias, M. y Uria, A. (2014, 26-27 de noviembre.). El cuerpo del docente en las prácticas pedagógicas del nivel inicial: revisión de la categoría de análisis disponibilidad corporal. [Ponencia]. II Jornadas Latinoamericanas de Investigadores/as en Formación en Educación. Evento organizado por el Instituto de Investigaciones en Ciencias de la Educación (IICE), Buenos Aires, Argentina. http://www.aapsicomotricidad.com.ar/assets/archivos\%20descarga/publicaciones/13-el-cuerpo-del-docente-en-las-practicas.pdf

Sparkes, A. (1996). Recordando los cuerpos de los profesores: momentos desde el mundo de la educación física. Revista de Educación, (311), 101-122. http://www.educacionyfp.gob.es/revista-de-educacion/numeros-revista-educacion/numeros-anteriores/1996/re311.html
Tarruella, N.L. y Rodríguez, L.B. (2008). La mirada en la organización corporal del/la docente. Revista Pilquen, 10(5), 1-5. https://dialnet.unirioja.es/servlet/ articulo? codigo $=3055556$

Vidal, F., Nicasio, J. y Pacheco, D. (2010). El burnout en los profesores. International Journal of Developmental and Educational Psychology, (1), 251-256. http://www. redalyc.org/articulo.oa?id=349832324027>

Villalba, R. (2015). Corporrelatos del yo docente: un inventario de experiencias contenidas en el cuerpo. [Tesis de maestría, Universidad Distrital Francisco José de Caldas]. Repositorio institucional UD (en versalitas). http://hdl.handle.net/11349/4765 